\title{
Effects of Neonatal Stress and Morphine on Murine Hippocampal Gene Expression
}

\author{
SANDRA E. JUUL, RICHARD P. BEYER, THEO K. BAMMLER, FEDERICO M. FARIN, AND CHRISTINE A. GLEASON
}

Department of Pediatrics [S.E.J., C.A.G.], Department of Environmental and Occupational Health Sciences [R.P.B., T.K.B., F.M.F.], University of Washington, Seattle, Washington 98195

\begin{abstract}
Critically ill preterm infants experience multiple stressors while hospitalized. Morphine is commonly prescribed to ameliorate their pain and stress. We hypothesized that neonatal stress will have a dose-dependent effect on hippocampal gene expression, and these effects will be altered by morphine treatment. Male C57BL/6 mice were exposed to five treatment conditions between postnatal d 5 and 9: 1) control, 2) mild stress + saline, 3) mild stress + morphine, 4) severe stress + saline, and 5) severe stress + morphine. Hippocampal RNA was extracted and analyzed using Affymetrix Mouse Gene 1.0 ST Arrays. Single gene analysis and gene set analysis were used to compare groups with validation by qPCR. Stress resulted in enrichment of gene sets related to fear response, oxygen carrying capacity, and NMDA receptor synthesis. Morphine down-regulated gene sets related to immune function. Stress + morphine resulted in enrichment of mitochondrial electron transport gene sets and downregulation of gene sets related to brain development and growth. We conclude that neonatal stress alone influences hippocampal gene expression, and morphine alters a subset of stress-related changes in gene expression and influences other gene sets. Stress + morphine show interaction effects not present with either stimulus alone. These changes may alter neurodevelopment. (Pediatr Res 69: 285-292, 2011)
\end{abstract}

$\mathrm{T}$ he effects of stress on health and development may be either positive or negative. For example, short-term acute stress can boost the immune system, but prolonged inescapable stress can have deleterious effects on learning, development, the immune system and may increase susceptibility to degenerative diseases (1-3). Preterm infants in the NICU are exposed to prolonged inescapable stress. On average, a 28 -wk gestation infant spends $10-12$ wk (approximately the last trimester of pregnancy) in a NICU. During this time of rapid brain development, they are separated from their mothers, handled repeatedly, exposed to multiple painful procedures, and they may also be mechanically ventilated, gavage fed, and exposed to repeated periods of oxidative stress. Neurodevelopmental outcomes following extreme prematurity remain poor, with moderate

Received June 28, 2010; accepted November 8, 2010.

Correspondence: Sandra Juul, M.D., Ph.D., Division of Neonatology, Department of Pediatrics, University of Washington, Box 356320, Seattle, WA 98195-6320; e-mail: sjuul@uw.edu

Supported by Grant R21DA022573 from NIH/NIDA and Grant P30HD002274 from NIH/NICHD.

Supplemental digital content is available for this article. Direct URL citations appear in the printed text and are provided in the HTML and PDF versions of this article on the journal's Web site (www.pedresearch.org). to severe impairment occurring in close to $50 \%$ of extremely LBW infants (4). Autism, attention deficit disorder, and school failure also occur more frequently in NICU survivors (5). Although some degree of impairment might be inevitable, it is likely that the stress and treatments these infants undergo impact neurologic outcome. Improved understanding of these factors will provide the basis of better treatments and subsequent improvement in outcomes.

Many preterm infants receive opiates for sedation or analgesia during their NICU stay. Treatment occurs during a period of intense brain development, with brain weight tripling during the last trimester of pregnancy (6). Neuronal differentiation, migration, and synapse formation are active processes through term gestation, as is glial proliferation (7). Complex neural networks form in regionally specific ways $(8,9)$, controlled by families of netrins, ephrins, semaphorins, and slits (10). In the rat, neonatal stress disrupts subsequent adult learning and maturation of the adrenal stress response (1-3). Morphine exposure may have additional consequences in the newborn, affecting both immune function and neurodevelopment (11-14). The combined effects of stress and morphine have not been well studied; however, they are of vital importance because the at-risk period when critically ill infants are exposed to morphine is also a time during which they are experiencing inescapable stress (15).

Brain development in the third trimester of human gestation generally corresponds to the first $2 \mathrm{wk}$ of postnatal life in mice (16). Therefore, use of neonatal rodents to model preterm brain development is reasonable, despite the differences in brain complexity. We developed a rodent model of neonatal stress that simulates many of the experiences of a hospitalized preterm infant. We previously reported short-term hippocampal gliosis (17) and long-term neurobehavioral effects of stress and morphine exposure in our mouse model of neonatal stress (18). To further investigate the mechanism by which these changes occur, we now hypothesize that repeated neonatal stress will have a

Abbreviations: CC, untreated controls; GSA, gene set analysis; MSM, mild stress + morphine; MSS, mild stress + saline; NPY, neuropeptide Y; SSM, severe stress + morphine; SSS, severe stress + saline; P, postnatal day; qPCR, quantitative PCR 
dose-dependent effect on hippocampal gene expression and that these effects will be altered by morphine treatment.

\section{MATERIALS AND METHODS}

Animals. Adult WT C57BL/6 mice were purchased (Harlan) and housed under a 12-h light-dark cycle with free access to food and water. All animal procedures were approved by the Animal Care and Use Committee at the University of Washington.

Treatment groups. Male mice were exposed to five treatment conditions between postnatal d (P)5 and P9 ( $n=3$ /group), with birth recorded as P1. Litters were culled to $n=7$ maximum per dam. The following groups were included: 1) untreated controls (CC), 2) mild stress + saline (MSS), 3) mild stress + morphine (MSM), 4) severe stress + saline (SSS), and 5) severe stress + morphine (SSM). Only males were used so as to eliminate sex-related genetic variability. CC animals underwent minimal handling on P5 and at killing on P9. All animals were killed on P9. Groups 2-5 received s.c. $10 \mu \mathrm{L}$ injections of either saline or morphine twice daily at $0800 \mathrm{~h}$ and $1530 \mathrm{~h}$. The morphine (Baxter) dose was $2 \mathrm{mg} / \mathrm{kg}$ in a $10-\mu \mathrm{L}$ volume and based on the daily average litter weight. This dose produces circulating morphine levels that approximate the range measured in human preterm infants given standard intermittent i.v. morphine boluses or continuous i.v. morphine infusion (19). Severely stressed pups (groups 4 and 5) were separated from the dam and isolated in individual containers within a veterinary warmer at $32^{\circ} \mathrm{C}$ from $0800 \mathrm{~h}$ until $1600 \mathrm{~h}$, thus experiencing both maternal and littermate separation. Pups were gavage fed with $50-150 \mu \mathrm{L}$ of rodent milk substitute at 1000, 1200, and $1400 \mathrm{~h}$ using a 24-gauge animal feeding needle (Popper \& Sons, New Hyde Park, NY). To simulate the oxidant stress of apnea, pups were exposed to hypoxia [ $100 \%$ nitrogen for $1 \mathrm{~min}$ followed by hyperoxia (100\% oxygen for $5 \mathrm{~min}$ ) twice daily $(0800$ and $1530 \mathrm{~h}$ )]. Mice were then returned to the dam with their concurrent unstressed littermates each evening and allowed to nurse overnight ad lib.

RNA methods. RNA from the right hippocampus was isolated (Cartagen Molecular Systems, San Carlos, CA). RNA quality was evaluated using an Agilent 2100 Bioanalyzer (Agilent Technologies, Inc., Palo Alto, CA) Only RNA samples with appropriate size distribution, quantity, and an A260:A280 ratio of $1.8-2.1$ were used for analysis.

Microarray processing. The manufacturer's protocols for the GeneChip platform by Affymetrix (Santa Clara, CA) were used. Methods included synthesis of first- and second-strand cDNAs, the purification of doublestranded cDNA, synthesis of cRNA by in vitro transcription, recovery and quantitation of biotin-labeled cRNA, fragmentation of this cRNA and subsequent hybridization to the microarray slide, posthybridization washings, and detection of the hybridized cRNAs using a streptavidin-coupled fluorescent dye. Hybridized Affymetrix arrays were scanned with an Affymetrix GeneChip 3000 scanner. Image generation and feature extraction were performed using Affymetrix GCOS software.

Statistical analysis and data normalization for Affymetrix mouse gene 1.0 ST arrays. Raw microarray data were processed with Bioconductor (20). Probes were normalized with robust multiarray (RMA) (21). From the normalized data, genes with significant evidence for differential expression were identified using the Bioconductor limma package (22). The $p$ values were calculated with a modified $t$ test in conjunction with an empirical Bayes method to moderate the standard errors of the estimated log-fold changes. The $p$ values were adjusted for multiplicity with the Bioconductor package qvalue (23) that allows for selecting statistically significant genes while controlling the estimated false discovery rate.

Single gene analysis. The experimental design, with the specific main effects and the interaction effects, that we wished to examine is shown in Figure 1 and Table 1. Figure 1 shows the basis for our comparisons and what specific questions they answer. We define 1) a stress-affected group of genes as the union of the mild and severe stress response genes (MSS-CC union with SSS-MSS), 2) a morphine-affected group of genes as the union of the morphine response genes in the context of mild and severe stress (MSM-MSS union with SSM-SSS), and 3) the interaction effects of severe-stress with morphine as genes that react differently to morphine in the presence of severe stress than with mild stress.

Table 1 outlines the mathematical considerations that we took into account when making group comparisons. For example, to answer the question "which genes increased by stress $\left(\beta_{1}+\beta_{2} \gg 1\right)$ does morphine affect?" we look for genes that satisfy the equation:

$$
\alpha_{1}+\alpha_{2}+\beta_{1}+\beta_{2}+\gamma_{1}+\gamma_{2}+\gamma_{3}+\gamma_{4} \sim 0 .
$$

Gene set analysis. To investigate categories of genes where the constituent genes show coordinated changes in expression over the experimental

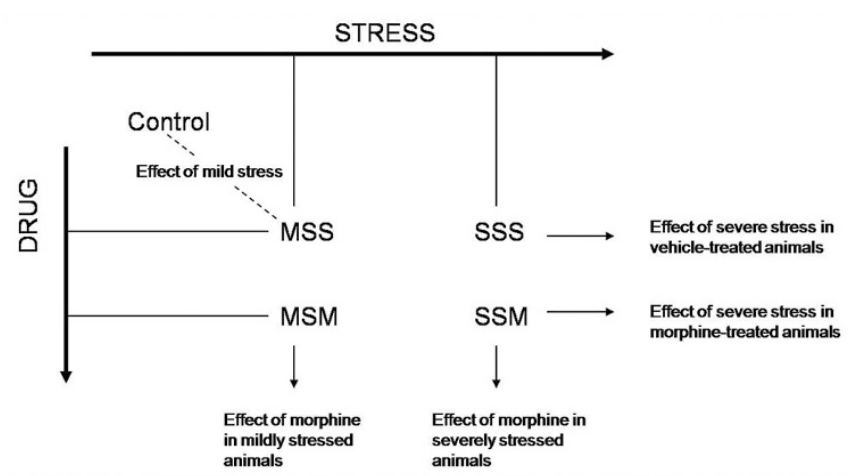

Figure 1. Stress morphine comparison groups. These comparisons allow us to answer the following questions: 1) What is the effect of mild stress? Answer: MSS-CC; 2) Is there a dose-dependent effect of stress? Answer: SSS-MSS; 3) How does severe stress affect morphine-treated animals? Answer: SSM-MSM; 4) How does morphine affect mildly stressed animals? Answer: MSM-MSS; 5) What does morphine do to severely stressed animals? Answer: SSM-SSS; and 6) Does morphine act different in severely stressed animals? Answer: stress $\times$ morphine interaction.

Table 1. Parametrization used for the microarray analysis

\begin{tabular}{|c|c|c|c|}
\hline & No stress & $\begin{array}{l}\text { Weight }+ \\
\text { injections }\end{array}$ & $\begin{array}{c}\text { Weight }+ \\
\text { injections }+ \\
\text { separation }+ \\
\text { hypoxia/hyperoxia }\end{array}$ \\
\hline $\begin{array}{l}\text { No } \\
\text { morphine }\end{array}$ & $\mu$ & $\mu+\beta 1$ & $\mu+\beta 1+\beta 2$ \\
\hline Vehicle & & $\begin{array}{l}\mu+\alpha 1+\beta 1 \\
\quad+\gamma 1\end{array}$ & $\begin{array}{l}\mu+\alpha 1+\beta 1+\gamma 1 \\
\quad+\beta 2+\gamma 3\end{array}$ \\
\hline $\begin{array}{l}\text { Vehicle }+ \\
\text { morphine }\end{array}$ & $\mu+\alpha 1+\alpha 2$ & $\begin{array}{l}\mu+\alpha 1+\beta 1 \\
\quad+\gamma 1+\alpha 2+\gamma 2\end{array}$ & $\begin{array}{l}\mu+\alpha 1+\beta 1+\gamma 1 \\
\quad+\alpha 2+\gamma 2+\beta 2+\gamma 3 \\
\quad+\gamma 4\end{array}$ \\
\hline
\end{tabular}

$\alpha_{1}$, effect of vehicle; $\alpha_{2}$, effect of morphine; $\beta_{1}$, effect of mild stress; $\beta_{2}=$ effect of severe stress; $\gamma_{1}$, interaction effect of vehicle; $\alpha_{1}$, with mild stress, $\beta_{1}$, probably zero; $\gamma_{2}$, interaction effect of morphine, $\alpha_{2}$, with $\beta_{1}+\gamma_{1}$, probably negligible; $\gamma_{3}$, interaction effect of $\alpha_{1}+\gamma_{1}$ with severe stress, $\beta_{2} ; \gamma_{4}$, interaction effect of $\alpha_{2}+\gamma_{2}$ and $\beta_{2}+\gamma_{3}$.

conditions, we used gene set analysis (GSA), a type of biological category analysis $(20,24)$. GSA software is available as R code $(24,25)$ (http:// www-stat.stanford.edu/ tibs/GSA/) to calculate separate gene set analyses. GSA considers all the genes in the experiment and allows for the identification of gene sets with strong cross correlation by boosting the signal-to-noise ratio, making it possible to detect modest changes in gene expression. The term "gene set" refers to genes that are grouped together based on function. For example, each gene ontology category (e.g. apoptosis) is a gene set. We used four gene set databases for GSA: biological process, molecular function, and cellular component from Gene Ontology (26) and the C2 gene set from the Molecular Signature Database (25) (http://www.broad.mit.edu/gsea/msigdb/msigdb_index.html.

Validation of microarray data using fluorogenic 5' nuclease-based assay and quantitative RT-PCR. RNA from separate, additional animals were used for validation. Quantitation of specific mRNA levels were determined as previously described (27) using fluorogenic 5' nuclease-based assays on an ABI 7900 Sequence Detection System (28).

\section{RESULTS}

Forty-seven genes were affected by mild stress, and 156 genes were affected by severe stress (up or down fold change $>1.3$-fold and $p<0.05$ ) including Dnajb6 (Hsp40 homolog), aminolevulinic acid synthase 2 (Alas2), and secreted phosphoprotein 1 (Sppl). Morphine had effects on three broad categories of genes: 1) morphine-responsive genes that were unaffected by stress $(n=92), 2)$ morphine- 
Table 2. Selected morphine-responsive genes*, unaffected by stress (92 genes)

\begin{tabular}{|c|c|c|c|c|c|}
\hline $\begin{array}{c}\text { Gene } \\
\text { symbol }\end{array}$ & Gene description & $\begin{array}{c}\text { MSM-MSS } \\
\left(\log _{2} \mathrm{FC}\right) \dagger\end{array}$ & $\begin{array}{c}\text { MSM-MSS } \\
p \text { value } \ddagger\end{array}$ & $\begin{array}{l}\text { SSM-SSS } \\
\left(\log _{2} \mathrm{FC}\right) \dagger\end{array}$ & $\begin{array}{c}\text { SSM-SSS } \\
p \text { value }\end{array}$ \\
\hline Accn 4 & Amiloride-sensitive cation channel 4 & 0.400 & 0.114 & 0.605 & 0.024 \\
\hline Aqp1 & Aquaporin 1 & 1.187 & $\mathbf{0 . 0 3 0}$ & 0.260 & 0.600 \\
\hline Avpr1a & Arginine vasopressin receptor $1 \mathrm{~A}$ & -0.704 & 0.024 & -0.160 & 0.570 \\
\hline Bex4 & Brain-expressed gene 4 & 0.015 & 0.901 & 0.389 & 0.006 \\
\hline Carhsp1 & Calcium-regulated heat stable protein 1 & 0.221 & 0.300 & 0.477 & $\mathbf{0 . 0 3 7}$ \\
\hline $\operatorname{Cdh} 22$ & Cadherin 22 & 0.026 & 0.861 & 0.405 & 0.016 \\
\hline Cldn2 & Claudin 2 & 0.744 & 0.029 & 0.245 & 0.430 \\
\hline Ctnnal1 & Catenin (cadherin-associated protein), alpha-like 1 & 0.415 & 0.021 & 0.137 & 0.396 \\
\hline Cux2 & Cut-like homeobox 2 & 0.653 & 0.013 & 0.007 & 0.977 \\
\hline Dlx5 & Distal-less homeobox 5 & 0.088 & 0.752 & 0.614 & 0.044 \\
\hline Dock10 & Dedicator of cytokinesis 10 & 0.122 & 0.445 & 0.410 & 0.027 \\
\hline Gabra4 & Gamma-aminobutyric acid (GABA-A) receptor, subunit alpha 4 & 0.101 & 0.385 & 0.392 & 0.004 \\
\hline Gad2 & Glutamic acid decarboxylase 2 & 0.231 & 0.424 & 0.609 & 0.0495 \\
\hline Gal & Galanin & 0.408 & 0.033 & 0.074 & 0.669 \\
\hline $\mathrm{Gh}$ & Growth hormone & -0.189 & 0.514 & -0.854 & 0.010 \\
\hline Glra2 & Glycine receptor, alpha 2 subunit & 0.171 & 0.414 & 0.441 & 0.049 \\
\hline Mpeg1 & Macrophage-expressed gene 1 & 0.017 & 0.870 & 0.384 & 0.003 \\
\hline Npy & Neuropeptide Y & 0.305 & 0.052 & 0.539 & 0.002 \\
\hline Reln & Reelin & -0.097 & 0.532 & -0.491 & 0.007 \\
\hline Rgs6 & Regulator of G-protein signaling 6 & 0.439 & 0.010 & 0.066 & 0.657 \\
\hline Tac1 & Tachykinin 1 & 0.083 & 0.860 & 1.374 & 0.012 \\
\hline Trh & Thyrotropin-releasing hormone & 0.700 & 0.029 & 0.275 & 0.350 \\
\hline
\end{tabular}

Columns 1 and 2 list gene symbols and gene descriptions. Columns 3 [MSM-MSS $\left.\left(\log _{2} \mathrm{FC}\right)\right]$ and 5 [SSM-SSS ( $\log _{2}$ FC)] provide the $\log _{2}$ transformed change in expression caused by mild-stress-morphine (MSM) treatment relative to the mild-stress-saline (MSS) treatment and the severe-stress-morphine (SSM) treatment relative to severe-stress-saline (SSS) treatment, respectively. Columns 4 (MSM-MSS $p$ value) and 6 (SSM-SSS $p$ value) list the $p$ values associated with the aforementioned contrasts.

* Morphine-responsive genes are defined as changing $>1.3$-fold (up or down) with $p<0.05$ in either the MSM-MSS or the SSM-SSS contrast.

$\dagger$ Fold change is provided as $\log _{2}$, positive numbers indicate gene was expressed at higher level in MSM vs MSS or SSM vs SSS, respectively, whereas negative numbers indicate the opposite, and bold values indicate a $>1.3$-fold absolute change.

\$ Bold values indicate $p<0.05$.

responsive genes that were also stress-responsive $(n=8)$, and 3 ) genes that responded only when both morphine and stress were present together, i.e. a morphine $\times$ stress interaction effect $(n=104)$. Morphine-responsive genes were defined as those which changed expression $>1.3$-fold (up or down) with $p<0.05$ in either the mild stress (MSM-MSS) or the severe stress (SSM-SSS) contrasts. A subset of morphine-responsive genes are shown in Table 2. In comparison, a subset of the interaction-specific genes is shown in Table 3. Examples of how treatment conditions affect gene expression are illustrated in Figure 2 using quantitative RT-PCR (qPCR). The relationship between genes affected by mild and severe stress is shown in the Venn diagram in Figure 3.

GSA allowed us to examine patterns of gene expression associated with each treatment group. Mild stress resulted in up-regulation of many gene sets related to immune function including the following: IFN $\gamma$ production, IL-1, 2, and 12 synthetic processes, defense response to Gram-positive bacterium, regulation of T-helper cell differentiation, positive regulation of cell killing, and leukocyte-mediated cytotoxicity. Other upregulated gene sets included positive regulation of systemic arterial blood pressure and glucocorticoid metabolic process. Down-regulated gene sets included those related to DNA and cellular maintenance.
When animals undergoing severe stress were compared with controls, gene sets consistent with stress such as multicellular organismal response to stress and fear response were overexpressed. However, unexpected were the many up-regulated gene sets related primarily to neurodevelopment and inflammation: dendrite morphogenesis, microtubule bundle formation, synaptic vessel exocytosis, cerebral cortex cell migration, axon regeneration, neurite development, wnt signaling pathway, ionotropic glutamate receptor signaling pathway, axon regeneration, nerve growth factor receptor signaling pathway, cell proliferation in forebrain, regulation of synapse structure and activity; IL-6 production, T-cell signaling pathway, and regulation of cytokine production (GSA $p<0.001$, false discovery rate $<0.001)$. Severe stress down-regulated gene sets related to glutamine family amino acid catabolic process, glutamate metabolic process, mismatch repair, nuclear mRNA splicing, phagocytosis, histone modification, DNA metabolic process, folic acid synthesis, and telomere maintenance. Supplementary Table $\mathrm{S} 1 A$ and $B$ (http:// links.lww.com/PDR/A66) gives more details of severe stress-related gene changes.

When comparing animals exposed to MSM with mildly stressed animals (MSS), there was down-regulation of 
Table 3. Selected genes responding only when stress and morphine are present together (104 genes total)

\begin{tabular}{|c|c|c|c|}
\hline $\begin{array}{l}\text { Gene } \\
\text { symbol }\end{array}$ & Gene description & $\begin{array}{l}\text { Stress and } \\
\text { morphine } \\
\text { interaction } \\
(\log 2 \mathrm{FC})^{*}\end{array}$ & $\begin{array}{c}\text { Stress and } \\
\text { morphine } \\
\text { interaction } \\
p \text { value }\end{array}$ \\
\hline $\mathrm{C} 1 \mathrm{ql3}$ & C1q-like 3 & 0.497 & 0.038 \\
\hline Calb1 & Calbindin-28K & 0.557 & 0.011 \\
\hline Dhrs7b & $\begin{array}{l}\text { Dehydrogenase/reductase (SDR } \\
\text { family) member 7B }\end{array}$ & 0.396 & 0.011 \\
\hline Dnahc3 & Dynein, axonemal, heavy chain 3 & -0.440 & 0.013 \\
\hline Dync1h1 & $\begin{array}{l}\text { Dynein cytoplasmic } 1 \text { heavy } \\
\text { chain } 1\end{array}$ & -0.385 & 0.005 \\
\hline Ephx2 & $\begin{array}{l}\text { Epoxide hydrolase } 2 \\
\text { cytoplasmic }\end{array}$ & 0.384 & 0.004 \\
\hline Fbn1 & Fibrillin 1 & -0.412 & 0.013 \\
\hline Hs6st2 & $\begin{array}{l}\text { Heparan sulfate } \\
\text { 6-O-sulfotransferase } 2\end{array}$ & 0.4345 & 0.012 \\
\hline Htr3a & $\begin{array}{l}\text { 5-Hydroxytryptamine (serotonin) } \\
\text { receptor } 3 \mathrm{~A}\end{array}$ & 0.591 & 0.018 \\
\hline Lrp1 & $\begin{array}{l}\text { Low-density lipoprotein } \\
\text { receptor-related protein } 1\end{array}$ & -0.445 & 0.012 \\
\hline Me3 & $\begin{array}{l}\text { Malic enzyme } 3 \text {, } \\
\text { NADP }(+) \text {-dependent, } \\
\text { mitochondrial }\end{array}$ & 0.401 & 0.035 \\
\hline Myo19 & Myosin XIX & -0.392 & 0.001 \\
\hline Nav1 & Neuron navigator 1 & -0.385 & 0.015 \\
\hline Pknox1 & $\mathrm{Pbx} /$ knotted 1 homeobox & 0.434 & 0.001 \\
\hline Rab11b & $\begin{array}{l}\text { RAB11B, member RAS } \\
\text { oncogene family }\end{array}$ & 0.392 & 0.019 \\
\hline $\operatorname{Rrm} 2 b$ & $\begin{array}{l}\text { Ribonucleotide reductase M2 B } \\
\text { (TP53 inducible) }\end{array}$ & 0.384 & 0.010 \\
\hline Sst & Somatostatin & 0.497 & 0.006 \\
\hline Syt5 & Synaptotagmin V & 0.379 & 0.014 \\
\hline Trhr & $\begin{array}{l}\text { Thyrotropin-releasing hormone } \\
\text { receptor }\end{array}$ & 0.487 & 0.046 \\
\hline Ttll11 & $\begin{array}{l}\text { Tubulin tyrosine ligase-like } \\
\text { family, member } 11\end{array}$ & 0.441 & 0.008 \\
\hline Txndc4 & $\begin{array}{l}\text { Thioredoxin domain containing } 4 \\
\text { (endoplasmic reticulum) }\end{array}$ & 0.381 & 0.019 \\
\hline Zfp239 & Zinc finger protein 239 & 0.380 & 0.009 \\
\hline
\end{tabular}

* Fold change is provided as $\log _{2}$, positive numbers indicate that the stress and morphine interaction effect increased gene expression, whereas a negative number indicates the opposite.

many gene sets related to immune function, particularly $\mathrm{T}$ and B-cell function and IL-1 and IL-12 synthesis. Overexpressed gene sets were not as thematic but included glutamine and dopamine metabolism, hyperosmotic response, and growth hormone secretion. Supplementary Table S2A and $B$ (http://links.lww.com/PDR/A66) gives more complete lists of gene sets whose expression is modified by MSM. Note that it is not possible to determine the effects of morphine in the absence of stress, because handling the animals to give injections is a form of stress. Therefore, the comparison MSM versus MSS provides the best estimation of the morphine effect alone.

In contrast to MSM, SSM positively enriched gene sets were related in large part to mitochondrial electron transport, oxygen transport, ATP synthesis, ATP-coupled electron transport, oxidative phosphorylation, cellular response to stress, regulation of dendrite morphogenesis, Notch signaling pathway, regulation of apoptosis, amyloid precursor metabolic process, and regulation of acute inflammatory response. Down-regulated gene sets in this comparison included many gene sets involving neuronal development: regulation of axon extension, synaptic vesicle exocytosis, hippocampal development, cerebral cortex cell migration, dendrite development, and several gene sets related to histone modification. Examples of these changes are shown in Figures. 4 and 5; Figure 4 depicts the effects of combined SSM on genes involved in microtubule bundle formation, dendrite development, and regulation of axonogenesis, Figure 5 shows genes involved in aerobic respiration, cellular response to stress, and ATP-coupled electron transport. Supplementary Table S3A and $B$ (http://links.lww.com/ PDR/A66) gives more complete lists of gene sets whose expression is modified by SSM.

We selected a subset of genes to validate by qPCR. Figure 6 shows the comparison of qPCR versus. microarray data. The 


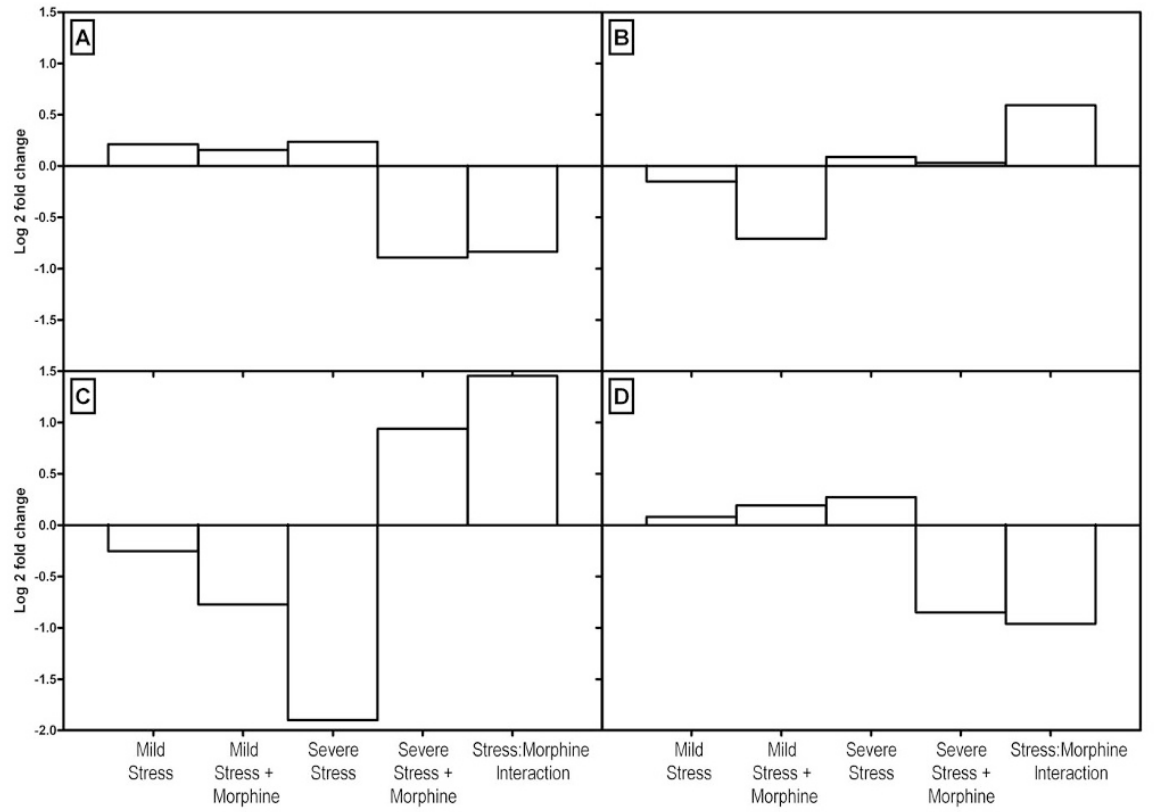

Figure 2. The qPCR of selected hippocampal genes by treatment condition. The qPCR results are normalized to GAPDH. The $x$ axis shows the conditions: mild stress, MSM, severe stress, SSM, and interaction effects of stress. The $y$ axis shows the log 2-fold change in gene expression. Expression of Nav1 (A), Wnt2 (B), Spp1 (C), and $\operatorname{Reln}(D)$ differ by treatment group, demonstrating that the effect of morphine is not necessarily predictable.

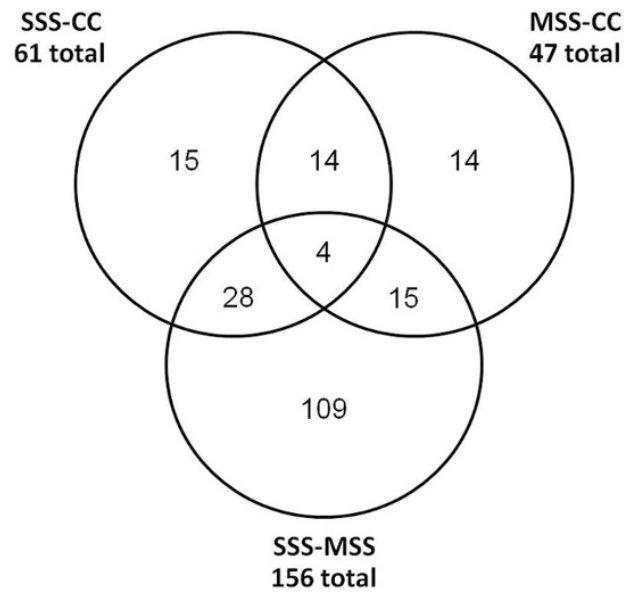

Figure 3. Venn diagram. This figure shows the relationship between genes affected by severe stress (SSS-CC), mild stress (MSS-CC), and the difference between mild and severe stress (SSS-MSS). Criteria for inclusion include fold $>1.3, p<0.05$.

correlation varies somewhat by condition, but overall the correlation is excellent.

\section{DISCUSSION}

As survival of the smallest infants improves, optimizing their neurodevelopmental potential becomes paramount. Although some degree of impairment following extreme prematurity might be inevitable, it is just as likely that over time, research will uncover pre- and postnatal treatments to protect the developing brain and improve outcomes. Most preterm infants receive analgesics and/or sedatives during their NICU stay, sometimes for weeks (29). During this period of neuronal network development, factors that influence neuronal excitation and inhibition result in profound changes in the balance of excitatory and inhibitory pathways and in cell survival (30). Clearly, both stress and opiate exposure have this potential. Further delineation of the individual and interactive effects of opiates with stress is of vital importance because the at-risk period when critically ill infants are exposed to morphine is concurrent with severe stress (15).

In this study, we show that repeated neonatal stress in mice has a dose-dependent effect on hippocampal gene expression and that the interactions of stress with morphine are complex. Although morphine does down regulate some stress-related changes in gene expression, this effect is not consistent across all stress-related genes. Depending on the gene in question and the degree of stress, treatment with morphine can have quite different and unpredictable effects. Figure 2 shows some examples of this: Wnt and Spp1 expression are changed in the presence of morphine, but the direction of change depends on the level of stress. These changes in hippocampal gene expression may significantly impact neurodevelopment, and subsequently, behavior.

Mild stress tended to increase genes related to immune function, blood pressure, and glucocorticoid expression, whereas decreasing those related to cell maintenance. Severe stress had less immune modulatory effects, but increased expression of gene sets having to do with neurodevelopment and inflammation, although down-regulating gene sets related to cellular repair such as telomere maintenance, mismatch repair, DNA metabolic process, folic acid synthesis, nuclear mRNA splicing, and histone modification.

Morphine exposure had different effects on hippocampal gene expression in the presence of mild versus severe stress. Treatment of MSM increased oxygen transport, mitochondrial membrane maintenance, and increased expression of specific gene sets related to neurodevelopment. Cholesterol, proteoglycan, and steroid synthesis were down-regulated. In contrast, treatment of SSM markedly 


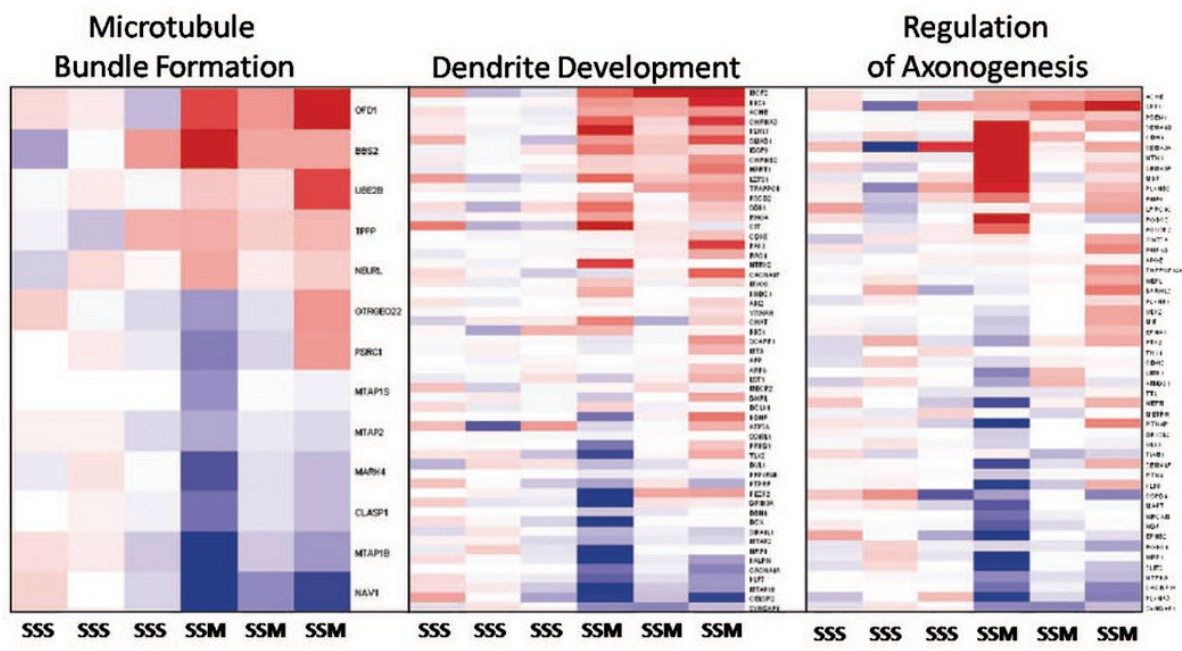

Figure 4. Neonatal stress and morphine treatment effects on selected neuronal activities. These composite heat maps show examples of three biological process gene sets related to neuronal development that differ by treatment group (GSA $p<0.0001$ ). Each row represents a specific gene in the gene set, and each column shows the pattern of RNA expression from an individual animal, arranged by treatment group labeled across the bottom. Individual animal variability can be seen, as well as group differences. Relative gene expression is denoted by color, with red illustrating gene expression that is higher than the mean in the control reference group and blue indicating the opposite and white indicating no change in expression. Expression levels for each gene are shown relative to the average expression of that gene in control animals. As the number of genes in the gene sets precludes adequate visibility, supplementary Table S4 (http://links.lww.com/PDR/A66) is provided listing the gene names in the same order as they appear in the heat maps (from top to bottom).

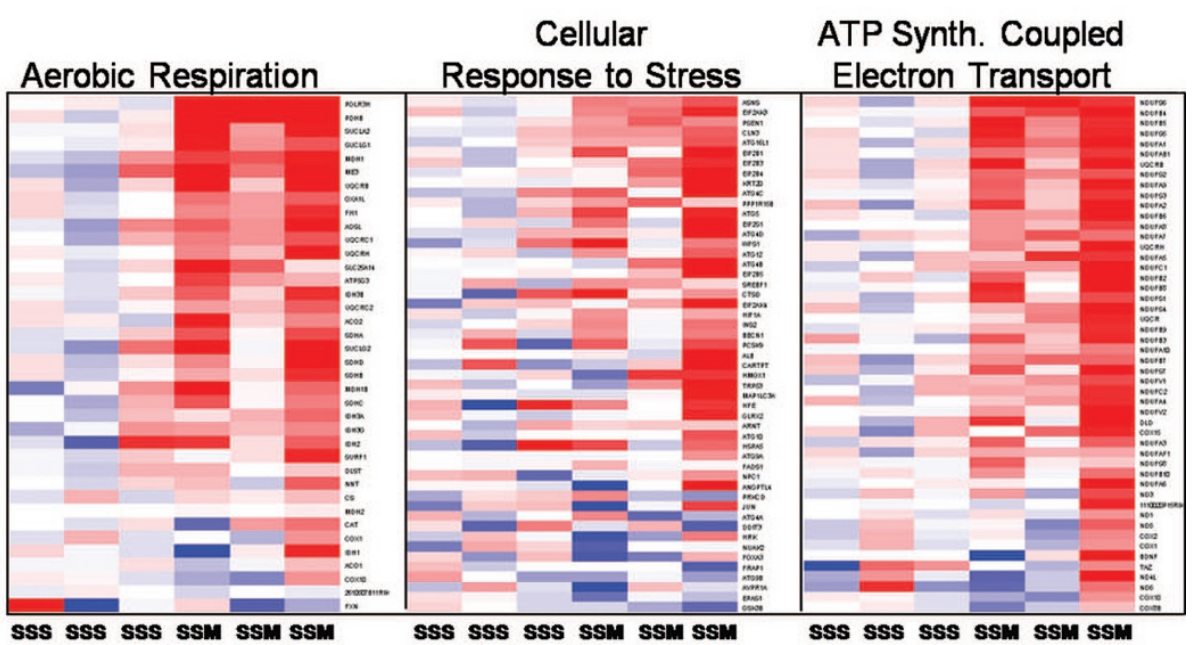

Figure 5. Neonatal stress and morphine treatment effects on cellular metabolism gene sets. Composite heat maps from severely stressed animals compared with severely stressed animals treated with morphine. Highlighted are gene sets related to energy metabolism. Although there is some variability from animal to animal (columns), and by individual gene (rows), the treatment of stress with morphine clearly increased gene expression in many of the genes in each family relating to aerobic respiration, cellular response to stress, and ATP synthesis-coupled electron transport (GSA $<0.0001)$. Because the number of genes in the gene sets precludes adequate visibility, supplementary Table S5 (http://links.lww.com/PDR/ A66) is provided listing the gene names in the same order as they appear in the heat maps (top to bottom). up-regulated expression in several gene sets regulating energy metabolism, oxidative phosphorylation, and aerobic respiration. Thus, it is apparent that the modulatory effects of morphine on hippocampal gene expression are dependent on the level of neonatal stress. Of particular concern, there was marked down-regulation in gene sets involving RNA processing and neurodevelopmental processes. These data suggest a mechanism by which decreased brain growth and other deleterious effects of morphine might be mediated.

This study is limited in that we could not assess the isolated effects of morphine, because morphine injections are by themselves stressful, and we also did not differentiate between the stressful stimuli of maternal separation, pain, formula feeding by gavage, and hypoxia/hyperoxia in the severely stressed group. These stimuli were combined to simulate the experiences preterm infants are exposed to. To assess isolated morphine effects, we com- pared mildly stressed morphine-treated animals with mildly stressed animals. This comparison showed up-regulation of estrogen metabolism, blood volume regulation, growth hormone synthesis, and glutamine metabolism, whereas immune function, urea cycle metabolism, and cell movement processes (microtubule polymerization) were downregulated.

Our data are consistent with previous work showing differential expression of Dnajbl (Hsp40) and glutamate receptor ionotropic kainate 2 (Grik2) in the C57BL6 striatum after morphine treatment (31). Our results with regard to neuropeptide Y (NPY) are also similar to a recent study showing $4 \mathrm{~d}$ morphine administration to mice caused an up-regulation of hypothalamic NPY gene expression (32). In addition, we found an up-regulation of galanin gene expression after morphine administration that is consistent with previous work demonstrating an increase in galanin 


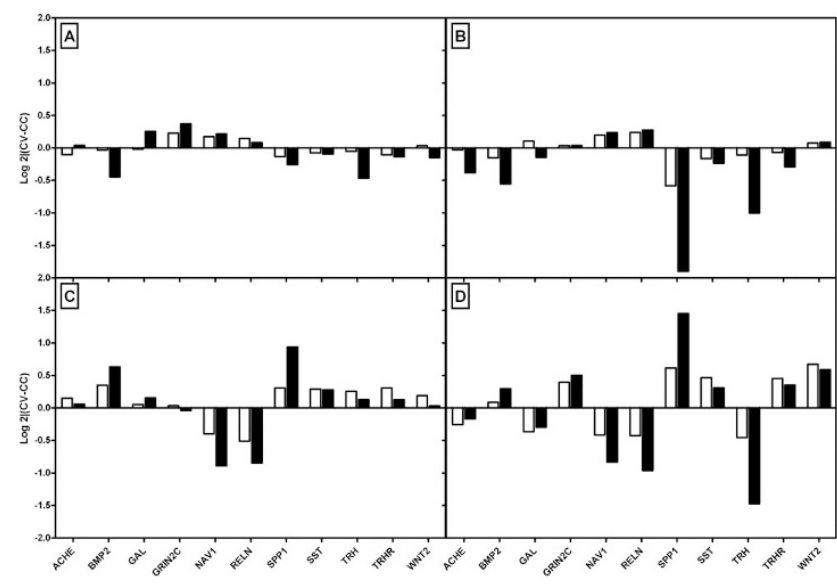

Figure 6. Validation of microarray results by qPCR. Microarray results were validated in 16 genes in all comparison conditions. A subset of 11 genes and conditions are shown in this figure. (A) Effects of mild stress, $(B)$ the effect of severe stress, $(C)$ effects of severe stress combined with morphine, and $(D)$ the effects of stress: morphine interaction. Microarray results are shown in clear bars and qPCR results in black for 11 genes. Gene names are listed on the $x$ axis. The $y$ axis shows the log 2-fold change in gene expression.

gene expression in mouse locus ceruleus and ventral tegmental region tissues after chronic morphine treatment (33). Erabi et al. (34) showed that neonatal stress (isolation) resulted in long-term changes the $I G F-I R$ and $I G F B P-2$ in the hippocampus in response to adulthood restraint stress, suggesting that epigenetic modifications occurred at the time of neonatal isolation. In our study, we found, but did not validate, changes in gene sets that relate to histone modification, which is a primary mechanism of epigenetic modification.

This study has provided a great deal of information about the neonatal hippocampal response to clinically relevant neonatal conditions: minor stress, severe stress including oxidative stress, and the interaction of morphine treatment with these conditions. We have validated differential expression of 16 genes of the 156 identified by microarray. We used RNA from a separate set of animals because we reasoned that if the findings were reproducible in these new animals, it not only validated the initial findings, vis a vis the validation of the technique, but also provided information regarding the reproducibility of the findings in animals treated with stress and/or morphine. Some of the differentially regulated genes are of uncertain importance, but others are likely to be of great importance and should be further studied. Many questions have been generated by this study. Why are genes and gene families related to energy metabolism up-regulated by the combination of stress and morphine? Is this beneficial or deleterious? What is the mechanism by which morphine and stress alter neurogenesis, synaptogenesis, axon formation, and neuronal differentiation? Are these effects long lasting? Are they dose dependent? Is there a dose of morphine which does not impair these functions, or perhaps alternative sedatives that do not have the same developmental effects? Most importantly, the findings from this study must make us question whether the current practice in the NICU is best for our patients. Although the treatment of unavoidable stress and pain in critically ill neonates is ethically mandated, the drugs we currently use may not, in fact, be effective in this population because of the immaturity of the nervous system (35), and they may also cause harm to the developing brain by interrupting normal developmental functions.

\section{REFERENCES}

1. Roma PG, Huntsberry ME, Riley AL 2007 Separation stress, litter size, and the rewarding effects of low-dose morphine in the dams of maternally separated rats. Prog Neuropsychopharmacol Biol Psychiatry 31:429-433

2. Mesquita AR, Pego JM, Summavielle T, Maciel P, Almeida OF, Sousa N 2007 Neurodevelopment milestone abnormalities in rats exposed to stress in early life. Neuroscience 147:1022-1033

3. Brunson KL, Chen Y, Avishai-Eliner S, Baram TZ 2003 Stress and the developing hippocampus: a double-edged sword? Mol Neurobiol 27:121-136

4. Marlow N, Wolke D, Bracewell MA, Samara M 2005 Neurologic and developmental disability at six years of age after extremely preterm birth. N Engl J Med 352:9-19

5. Johnson S, Hollis C, Kochhar P, Hennessy E, Wolke D, Marlow N 2010 Autism spectrum disorders in extremely preterm children. J Pediatr 156:525-531

6. Guihard-Costa AM, Larroche JC 1990 Differential growth between the fetal brain and its infratentorial part. Early Hum Dev 23:27-40

7. de Graaf-Peters VB, Hadders-Algra M 2006 Ontogeny of the human central nervous system: what is happening when? Early Hum Dev 82:257-266

8. Huttenlocher PR, Dabholkar AS 1997 Regional differences in synaptogenesis in human cerebral cortex. J Comp Neurol 387:167-178

9. Mrzljak L, Uylings HB, Van Eden CG, Judas M 1990 Neuronal development in human prefrontal cortex in prenatal and postnatal stages. Prog Brain Res $85: 185-222$

10. Dickson BJ 2002 Molecular mechanisms of axon guidance. Science 298:1959-1964

11. Saurer TB, Ijames SG, Carrigan KA, Lysle DT 2008 Neuroimmune mechanisms of opioid-mediated conditioned immunomodulation. Brain Behav Immun 22:89-97

12. Eisch AJ, Barrot M, Schad CA, Self DW, Nestler EJ 2000 Opiates inhibit neurogenesis in the adult rat hippocampus. Proc Natl Acad Sci U S A 97:7579-7584

13. Arguello AA, Harburg GC, Schonborn JR, Mandyam CD, Yamaguchi M, Eisch AJ 2008 Time course of morphine's effects on adult hippocampal subgranular zone reveals preferential inhibition of cells in $\mathrm{S}$ phase of the cell cycle and a subpopulation of immature neurons. Neuroscience 157:70-79

14. Handelmann GE, Dow-Edwards D 1985 Modulation of brain development by morphine: effects on central motor systems and behavior. Peptides 6:29-34

15. Simons SH, van Dijk M, Anand KS, Roofthooft D, van Lingen RA, Tibboel D 2003 Do we still hurt newborn babies? A prospective study of procedural pain and analgesia in neonates. Arch Pediatr Adolesc Med 157:1058-1064

16. Clancy B, Kersh B, Hyde J, Darlington RB, Anand KJ, Finlay BL 2007 Web-based method for translating neurodevelopment from laboratory species to humans. Neuroinformatics 5:79-94

17. Vien TN, Gleason CA, Hays SL, McPherson RJ, Chavkin C, Juul SE 2009 Effects of neonatal stress and morphine on kappa opioid receptor signaling. Neonatology 96:235-243

18. Boasen JF, McPherson RJ, Hays SL, Juul SE, Gleason CA 2009 Neonatal stress or morphine treatment alters adult mouse conditioned place preference. Neonatology 95:230-239

19. McPherson RJ, Gleason C, Mascher-Denen M, Chan M, Kellert B, Juul SE 2007 A new model of neonatal stress which produces lasting neurobehavioral effects in adult rats. Neonatology 92:33-41

20. Gentleman RC, Carey VJ, Bates DM, Bolstad B, Dettling M, Dudoit S, Ellis B, Gautier L, Ge Y, Gentry J, Hornik K, Hothorn T, Huber W, Iacus S, Irizarry R, Leisch F, Li C, Maechler M, Rossini AJ, Sawitzki G, Smith C, Smyth G, Tierney L, Yang JY, Zhang J 2004 Bioconductor: open software development for computational biology and bioinformatics. Genome Biol 5:R80

21. Irizarry RA, Gautier L, Cope L 2003 An R package for analyses of affymetrix oligonucleotide arrays. In: Parmigiani G, Garrett E, Irizarry R, Zeger S (eds) The Analysis of Gene Expression Data. Springer, London, pp 102-119

22. Smyth GK 2004 Linear models and empirical bayes methods for assessing differential expression in microarray experiments. Stat Appl Genet Mol Biol 3:3

23. Tusher VG, Tibshirani R, Chu G 2001 Significance analysis of microarrays applied to transcriptional responses to ionizing radiation. Proc Natl Acad Sci U S A 98:5116-5121

24. Efron B, Tibshirani R 2007 On testing the significance of sets of genes. Ann Appl Stat $1: 107-129$

25. Subramanian A, Tamayo P, Mootha VK, Mukherjee S, Ebert BL, Gillette MA Paulovich A, Pomeroy SL, Golub TR, Lander ES, Mesirov JP 2005 Gene set enrichment analysis: a knowledge-based approach for interpreting genome-wide expression profiles. Proc Natl Acad Sci U S A 102:15545-15550

26. Camon E, Magrane D, Barrell V, Lee E, Dimmer D, Maslen JB, Harte N, Lopez R, Apweiler R 2004 The Gene Ontology annotation (GOA) database: sharing knowledge in Uniprot with Gene Ontology. Nucleic Acids Res 32:D262-D266 
27. Juul SE, Beyer RP, Bammler TK, McPherson RJ, Wilkerson J, Farin FM 2009 Microarray analysis of high-dose recombinant erythropoietin treatment of unilateral brain injury in neonatal mouse hippocampus. Pediatr Res 65:485-492

28. Díaz D, Krejsa CM, White CC, Keener CL, Farin FM, Kavanagh TJ 2001 Tissue specific changes in the expression of glutamate-cysteine ligase mRNAs in mice exposed to methylmercury. Toxicol Lett 122:119-129

29. Anand KJ, Hall RW, Desai N, Shephard B, Bergqvist LL, Young TE, Boyle EM, Carbajal R, Bhutani VK, Moore MB, Kronsberg SS, Barton BA 2004 Effects of morphine analgesia in ventilated preterm neonates: primary outcomes from the NEOPAIN randomised trial. Lancet 363:1673-1682

30. Macrì S, Wurbel H 2006 Developmental plasticity of HPA and fear responses in rats: a critical review of the maternal mediation hypothesis. Horm Behav 50:667-680

31. Korostynski M, Piechota M, Kaminska D, Solecki W, Przewlocki R 2007 Morphine effects on striatal transcriptome in mice. Genome Biol 8:R128
32. Anghel A, Jamieson CA, Ren X, Young J, Porche R, Ozigbo E, Ghods DE, Lee ML, Liu Y, Lutfy K, Friedman TC 2010 Gene expression profiling following short-term and long-term morphine exposure in mice uncovers genes involved in food intake. Neuroscience 167:554-566

33. McClung CA, Nestler EJ, Zachariou V 2005 Regulation of gene expression by chronic morphine and morphine withdrawal in the locus ceruleus and ventral tegmental area. J Neurosci 25:6005-6015

34. Erabi K, Morinobu S, Kawano K, Tsuji S, Yamawaki S 2007 Neonatal isolation changes the expression of IGF-IR and IGFBP-2 in the hippocampus in response to adulthood restraint stress. Int J Neuropsychopharmacol 10:369-381

35. Carbajal R, Lenclen R, Jugie M, Paupe A, Barton BA, Anand KJ 2005 Morphine does not provide adequate analgesia for acute procedural pain among preterm neonates. Pediatrics 115:1494-1500 